Research Article

\title{
Classification of Electrocardiogram of Congenital Heart Disease Patients by Neural Network Algorithms
}

\author{
Yongjie Yuan $\mathbb{D}^{1},{ }^{1}$ Yongjun Zhang $\mathbb{D},{ }^{1}$ Junyuan Wang $\mathbb{D}^{2}$ and Ping Fang $\mathbb{D}^{2}$ \\ ${ }^{1}$ Department of Cardiac Function, \\ The First Affiliated Hospital of Wannan Medical College (Yijishan Hospital of Wannan Medical College), Wuhu 241001, \\ Anhui, China \\ ${ }^{2}$ Department of Cardiovascular Medicine, \\ The First Affiliated Hospital of Wannan Medical College (Yijishan Hospital of Wanan Medical College), Wuhu 241001, \\ Anhui, China \\ Correspondence should be addressed to Yongjie Yuan; yuanyongjie@wnmc.edu.cn
}

Received 16 June 2021; Accepted 16 August 2021; Published 31 August 2021

Academic Editor: Gustavo Ramirez

Copyright ( $\odot 2021$ Yongjie Yuan et al. This is an open access article distributed under the Creative Commons Attribution License, which permits unrestricted use, distribution, and reproduction in any medium, provided the original work is properly cited.

\begin{abstract}
The study intended to explore the effect of different neural network algorithms in the electrocardiogram (ECG) classification of patients with congenital heart disease (CHD). Based on the single convolutional neural network (CNN) ECG algorithm and the recurrent neural network (RNN) ECG algorithm, a multimodal neural network (MNN) ECG algorithm was constructed utilizing the MIT-BIH database as training set and test set. Furthermore, the MNN ECG algorithm was optimized to establish an improved MNN (IMNN) algorithm, which was applied to the diagnosis of CHD patients. The CHD patients admitted between August 2016 and August 2019 were selected for analysis to compare the classification effect and accuracy rate of IMNN, MNN, CNN ECG, and RNN ECG algorithms. It was found that the RNN ECG algorithm had higher classification sensitivity and true positive rate in terms of normal or bundle (NB) branch block beat, supraventricular abnormal (SA) rhythm, abnormal ventricular (AV) beat, and fusion beat $(\mathrm{FB})$ than the CNN ECG algorithm $(P<0.05)$, and the classification sensitivity and true positive rate of IMNN algorithm in the four aspects were significantly higher than those of MNN algorithm $(P<0.05)$. The classification accuracy of CNN ECG algorithm and RNN ECG algorithm was above 98\%, while that of MNN algorithm and IMNN algorithm was better than that of CNN ECG algorithm and RNN ECG algorithm, and the accuracy rate can reach $98.5 \%$ or more. Moreover, the accuracy rate of the IMNN algorithm can reach more than $98 \%$. In conclusion, IMNN not only has a good classification ability in the simulated environment but also performs well in the actual environment, which is worthy of clinical promotion.
\end{abstract}

\section{Introduction}

The heart is a vital organ. Heart disease is the number one killer threatening human health in the world today, and CHD is the most common type of congenital malformations, accounting for about $28 \%$ of various congenital malformations $[1,2]$. The incidence of CHD cannot be underestimated, accounting for $0.4 \%$ to $1 \%$ of living infants. It means that there are 150,000 to 200,000 new patients with $\mathrm{CHD}$ every year in China. Arrhythmia is the most common manifestation of CHD [3]. Once an arrhythmia occurs, it interferes with the heart's ability to pump blood and may cause sudden loss of heart function or cardiac arrest. ECG signal is one of the most effective clinical medical bio-signals for predicting heart disease. It is easy to detect and can clearly reflect the regularity of heartbeat fluctuations, so as to achieve effective diagnosis and prediction of heart disease [4]. At present, there are two main methods of electrocardiogram classification, namely, the classification method based on waveform shape and the classification method based on waveform characteristics. The classification method based on waveform shape needs to extract feature waveform. Then, medical classification rules are used to judge it. However, the classification method based on waveform shape requires high ECG quality, poor anti-interference ability and high recognition accuracy of each waveform feature point, so it is not suitable for dynamic ECG processing. The classification method based on 
waveform characteristics is the most widely used ECG classification and recognition method at present, and it can be combined with the deep neural network to make the overall classification accuracy higher $[5,6]$. At present, the clinical recognition of ECG signals mostly adopts the method of manual analysis by clinicians, but the diagnosis process is time-consuming and laborious, and the accuracy of the diagnosis results will be different due to the doctor's professional level. Realizing accurate, convenient, and fast automatic ECG signal recognition technology is the goal set by domestic and foreign scholars [7]. With the advancement of deep learning models, people have paid more attention to the direct classification of ECG signals using CNN models in recent years [8]. Among many deep learning models, multimodal neural network can obtain more comprehensive features, improve model robustness, and ensure that the model can still work effectively in the absence of some modes. Therefore, it is feasible to apply it in medicine.

Therefore, on the basis of neural network, a variety of neural networks were analyzed. Besides, a multimodal neural network was constructed to study its role in ECG classification and ECG analysis of CHD patients.

\section{Methods and Materials}

2.1. ECG Signal Classification of Single CNN. To explore the ability of CNN to extract features and classify ECG signals in the ECG signal dataset, a CNN was first designed, the structure of which is shown in Figure 1. The convolution model had three one-dimensional convolutional layers, three one-dimensional pooling layers, a fully connected layer, and a softmax output layer.

In the convolutional network, the input is a one-dimensional feature vector of length $L Y=\left[y_{1}, y_{2}, y_{3}\right.$, $\left.\ldots, y_{L}\right]$, the convolutional layer of the first layer is composed of $X$ convolution kernels, where the size of each convolution kernel is $1 \times Z$, and the coefficient of the convolution kernel is $w_{i} \in R^{S}, i=1,2,3, \ldots, I$. The output of the convolutional layer is $o=\left[o_{1}, o_{2}, o_{3}, \ldots, o_{i}\right]$, and the expression of $o_{i}$ is as follows:

$$
o_{i}=\operatorname{ReLU}\left(w_{i} \cdot y+b_{k}\right),
$$

where ReLU represents the nonlinear activation function and $b_{k}$ is the offset corresponding to the convolution kernel. Generally speaking, the number of hidden neurons contained in each layer should be much larger than the dimension of the input feature vector. After the calculation of the convolutional layer, the image does not appear to be obviously smaller, and the feature particles of the image are still scattered. On this basis, a pooling layer is added after the convolutional layer. It can ensure that the data are reduced in dimensionality on the basis of data translation with no deformation. Deep CNN is often composed of multiple convolutional pooling layers, multiple fully connected layers, and a softmax output layer. Assuming that the network has $N$ layers, $N_{m}$ is the fully connected layer, the $N_{n}$ th layer is the final output layer, and the number of output units is the final classification category $K$, then the whole calculation process is as follows:

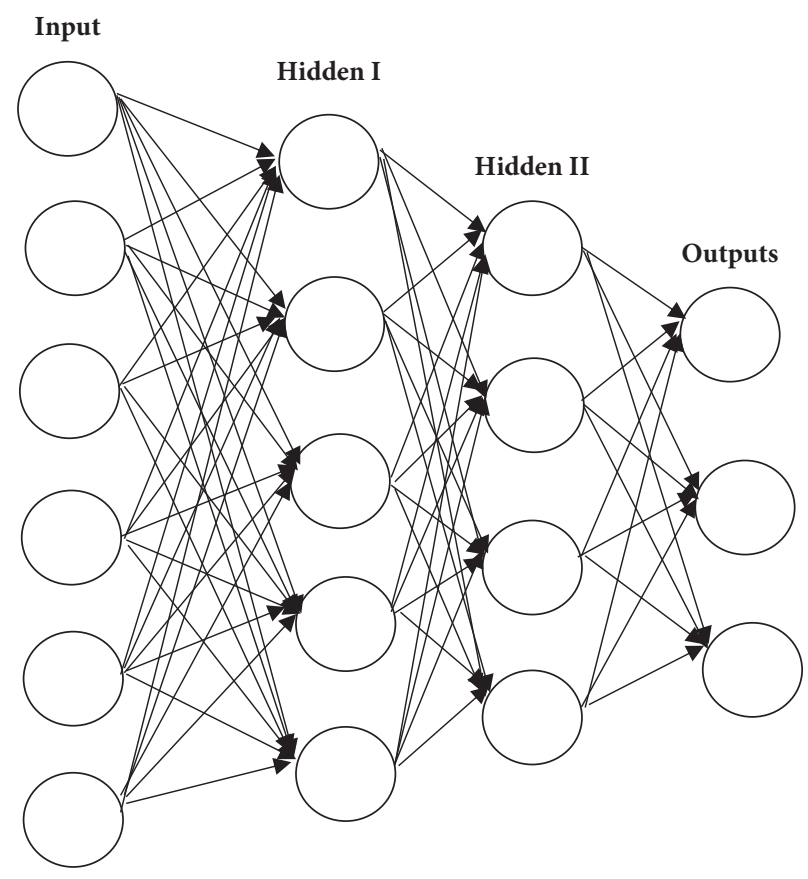

Figure 1: CNN structure.

$$
\begin{aligned}
o_{i} & =f\left(w^{i} o^{i}+b^{i}\right), \quad N_{m}<i<N_{n}, \\
a^{N n} & =w^{i} o^{i}+b^{i}, \\
P(t \mid x) & =\operatorname{softmax}\left(a^{N n}\right)=\frac{\exp \left(a_{t}^{N n}\right)}{\sum_{t=1}^{K} \exp \left(a_{t}^{N n}\right)}, \quad t=1,2,3, \ldots, K,
\end{aligned}
$$

where $o^{i}$ is the output of the $i$ th layer, $x^{i}$ and $y^{i}$ are relevant parameters that the network needs to learn, $a^{N n}$ is the value before the last output layer is activated, and $P(t \mid x)$ is the posterior probability that belongs to category $T$, which is judged by $\mathrm{CNN}$ after inputting $X$. Convolutional layer 1 of the single CNN contains 6 filters with a size of $1 \times 10$. Convolutional layer 2 contains 6 filters with a size of $1 \times 10$. Convolutional layer 3 contains 128 filters with a size of $1 \times 10$. Pooling layer 1 contains 6 filters of $1 \times 2$, pooling layer 2 contains 6 filters of $1 \times 2$, and pooling layer 3 contains 128 filters of the size of $1 \times 2$. There are 1024 filters in the fully connected layer.

2.2. ECG Signal Classification of Single RNN. RNN itself has certain advantages, especially the variant structure of RNN long short-term memory (LSTM) network. It is a special form of RNN which has three control units of the output gates, input gates, and forget gates. Bidirectional LSTM (BLSTM) further upgrades the LSTM network, so that the input at each moment comes from the information transmitted by the bank layer in the two directions. The network simultaneously combines the output of the front and back hidden layers and obtains the final output at each moment. Therefore, BLSTM was adopted for modeling and analysis, and its structure is shown in Figure 2. 


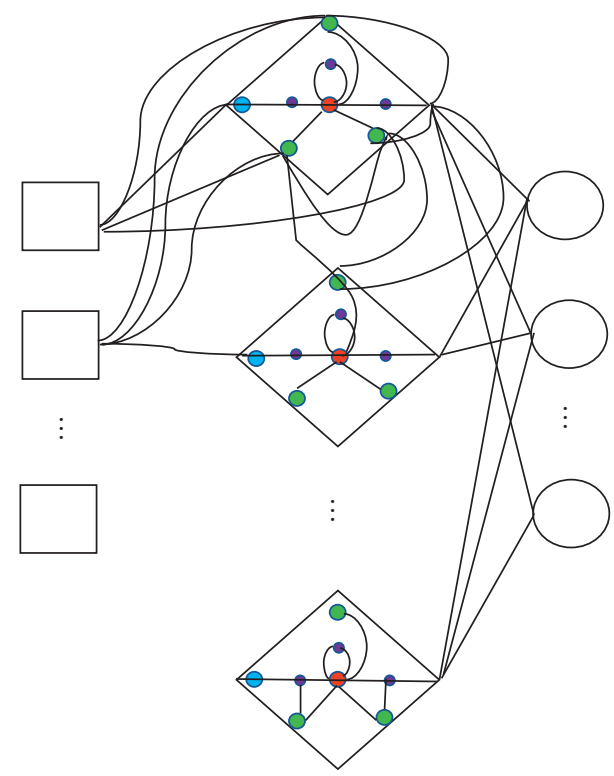

FIgURE 2: Bidirectional RNN.

When the two-dimension RNN performs forward calculations, it only shows the traditional one-way LSTM network. The forward calculation needs to be associated with the input data before the current time. The calculation equation for network forward is as follows:

$$
\vec{h}_{t}=W_{x h} x_{t}+W_{\overrightarrow{h h}} \vec{h}_{t-1}+a \vec{h}
$$

In the reverse calculation, the data to be input in the future are associated, and the network reverse calculation equation is as follows:

$$
\overleftarrow{h}_{t}=W_{x \overleftarrow{h}} x_{t}+W_{\overleftarrow{h} \overleftarrow{h}} \overleftarrow{h}_{t-1}+a_{h}
$$

The LSTM networks in the front and back directions will not be connected to each other, and the respective network states are maintained and guaranteed. On this basis, the network status in different directions is calculated on the output layer, and the calculation equation for the entire network is as follows:

$$
y_{t}=W_{\vec{h}^{y}} \vec{h}_{t}+W_{\overleftarrow{h}}^{y} \overleftarrow{h}_{t}+a_{y}
$$

\subsection{ECG Signal Classification of Multimodal Neural} Network. Because CNN and RNN have different advantages in the recognition and classification of ECG signals, a combined network structure was further designed on this basis. The last layer of the combined network was the feature fusion layer, and it spliced the spatial features obtained by CNN with the time sequence features obtained by BLSTM. Then, these features were sent to the final output layer for classification. The calculation of improved fusion feature network is as follows, where the input length is $L$ one-dimensional feature vector $A=\left[a_{1}, a_{2}, a_{3}, \ldots, A_{L}\right]$.

$$
h_{\mathrm{FFL}}=W_{\mathrm{FFL}}\left(\begin{array}{c}
a_{\mathrm{CNN}}^{L} \\
a_{\mathrm{BLSTM}}
\end{array}\right)+b_{\mathrm{FFL}}
$$

in which $h_{\mathrm{FFL}}$ is the hidden state information output by the $\mathrm{CNN}$ in the combined neural network, $a_{\mathrm{CNN}}^{L}$ is a set of effective ECG features extracted from the input ECG data by the network, and $a_{\mathrm{BLSTM}}$ is formed by splicing $x$ output from a set of RNBLSTM ECG signals at all time nodes. It can reflect the abnormal change of ECG signals to a greater extent in the time dimension. However, the feature fusion layer at this stage is only splicing in the direction of a onedimensional vector, and there will be certain redundant features between the morphological features extracted by CNN and the timing features extracted by RNN. Simply splicing into a fusion feature vector will cause redundancy in the number of features, slow down the network training speed, and affect the final classification effect of the network. Therefore, certain improvements were made on this basis in the study.

\subsection{ECG Signal Classification of Improved Multimodal Neural} Network. In this study, the attention mechanism (AM) neural network was combined with the multimodal neural network to make corresponding improvements, and the AM model is shown in Figure 3.

The decoding process of the attention model is as follows.

$$
\begin{aligned}
p\left(y_{i} \mid\left\{y_{1}, y_{2}, y_{3}, \ldots, y_{i-1}\right\}, x\right) & =f\left(y_{i-1}, s_{i}, a_{i}\right), \\
s_{i} & =g\left(s_{i-1}, y_{i-1}, a_{i}\right),
\end{aligned}
$$

where $a_{i}$ is the counted attention, whose function is to associate the output with the related input. The correlation degree between the current output and all inputs $b_{i j}$ is calculated as follows:

$$
a_{i}=\sum_{j=1}^{T_{x}} b_{i j} h_{j}
$$

where $u_{j}$ is the hidden layer information state of the encoder input at the $j$ th position and weight $b_{i j}$ is defined as follows according to equation (8):

$$
\begin{aligned}
& b_{i j}=\frac{\exp \left(e_{i j}\right)}{\sum_{k=1}^{T_{x}} \exp \left(e_{i k}\right)}, \\
& e_{i j}=b\left(s_{i-1}, h_{j}\right),
\end{aligned}
$$

where $e_{i j}$ is a prefeedback neural network. In the neural network, the attention module is usually an additional neural network that can give different weights to different parts of the input. Target data classification is more sensitive, which can effectively improve system performance in natural ways.

2.5. The Classification Evaluation Criteria. The MIT-BIH database provided by the Massachusetts Institute of Technology was selected as the experimental dataset. The data 


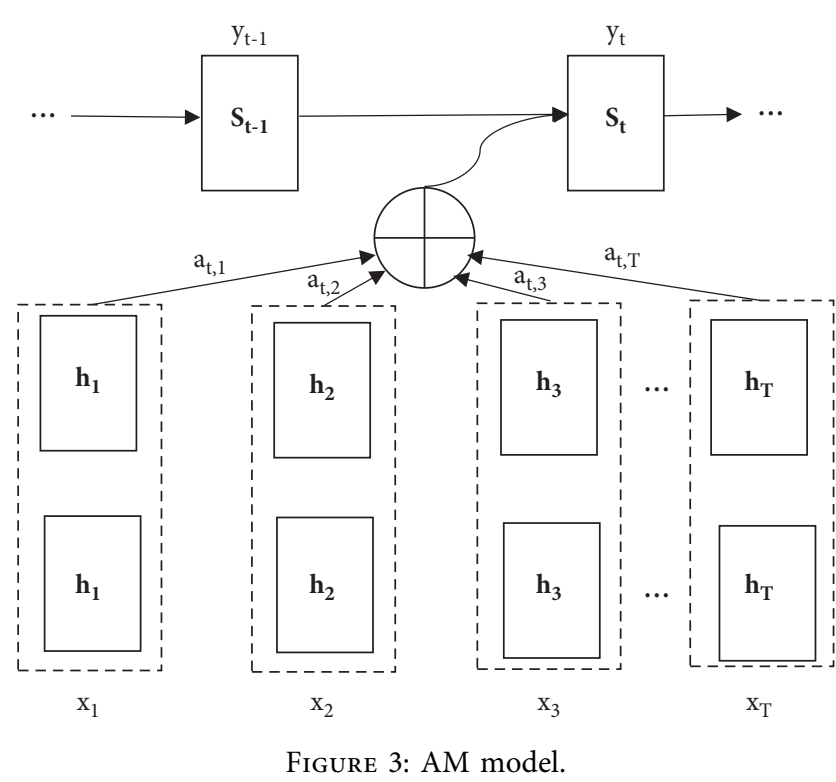

used in the experiment were randomly selected. The database consisted of 48 marked records and was divided into two groups. The first set of data contained 23 ECG records randomly selected from the ECG data, and the other group of data contained 25 ECG records that appeared rarely in practice but were clinically important. Each record took about 30 minutes, which was composed of two-way lead signals, and the signal sampling rate was $360 \mathrm{~Hz}$. The algorithm in this study was based on single-lead data, so only 45 recorded II-lead heartbeats were selected for the experiment. To make the classification results more standard, the ECG beats were classified as follows: normal or bundle branch block beat (NB), supraventricular abnormal rhythm (SA), abnormal ventricular beat (AV), fusion beat (FB), and uncategorized beats (UC).

2.6. Research Subjects. A total of 127 patients with $\mathrm{CHD}$ were selected to receive examinations in the hospital from August 2016 to August in 2019. They were diagnosed as CHD after ultrasound examination. The ultrasound examination is shown in Figure 4. Figures 4(a) and 4(b) show color Doppler ultrasound images of patients diagnosed as $\mathrm{CHD}$, respectively. On this basis, the same experienced professional doctor collected the single-lead ECG signals of all patients, preprocessed all the collected ECGs after the collection, and input all the data into the neural network for classification after preprocessing. All patients signed informed consent forms, and this study obtained permission from the ethics committee of the hospital.

2.7. Statistical Analysis. SPSS 22.0 was adopted for statistical analysis, and percentages were adopted to express the counting results. The $t$ test was adopted to compare the two groups, and $P<0.05$ meant obvious difference.

\section{Results}

3.1. Classification Comparison between Single CNN and Single $R N N$. The comparison of classification based on single CNN ECG algorithm and classification based on RNN ECG algorithm is shown in Figure 5. In contrast with the single $\mathrm{CNN}, \mathrm{RNN}$ had higher classification sensitivity and true positive rate in terms of NB, SA, AV, FB, and $\mathrm{UC}(P<0.05)$. In terms of classification accuracy, the classification accuracy of both was good $(P>0.05)$.

3.2. Comparison of Training Results. The experiments were all carried out with GTX1060 graphics card. The multimodal neural network ECG algorithm and the improved multimodal neural network ECG algorithm both used 82,600 ECG data for training and used the remaining 21,000 ECG data to test the corresponding model. The relationship between the loss value and the number of iterations of the multimodal neural network ECG algorithm is shown in Figure 6, and that of the improved multimodal neural network ECG algorithm is shown in Figure 7. The results show that the loss value of the improved multimode neural network ECG algorithm was obviously smaller.

3.3. Comparison of Classification Results. The classification of the multimodal neural network ECG algorithm and the improved one is shown in Figure 8. In contrast with the multimodal neural network, the improved one had higher classification sensitivity and true positive rate in terms of $\mathrm{NB}, \mathrm{SA}, \mathrm{AV}, \mathrm{FB}$, and UC $(P<0.05)$.

The accuracy of single CNN ECG algorithm, RNN ECG algorithm, multimodal neural network ECG algorithm, and improved multimodal neural network ECG algorithm was further compared, and the results are shown in Figure 9. The results showed that the classification results of the multimodal neural network ECG algorithm and the improved one were obviously better than the classification results of the single CNN ECG algorithm and the RNN ECG algorithm, and the single CNN ECG algorithm had the worst classification effect.

3.4. Actual Classification Results of CHD Patients. The actual clinical data were applied to classify CHD mixed with ECG, and the classification was carried out using the multimodal neural network ECG algorithm and the improved one (Figure 10). The results obtained in practice were similar to simulations. The classification accuracy of the improved multimodal neural network ECG algorithm was obviously higher than that of the multimodal neural network ECG algorithm, and the accuracy rate was higher than $98 \%$.

\section{Discussion}

In the automatic analysis of ECG signals at this stage, the characteristic detection of heartbeat beats is crucial because it influences a lot for obtaining heart activity [9]. Performing 


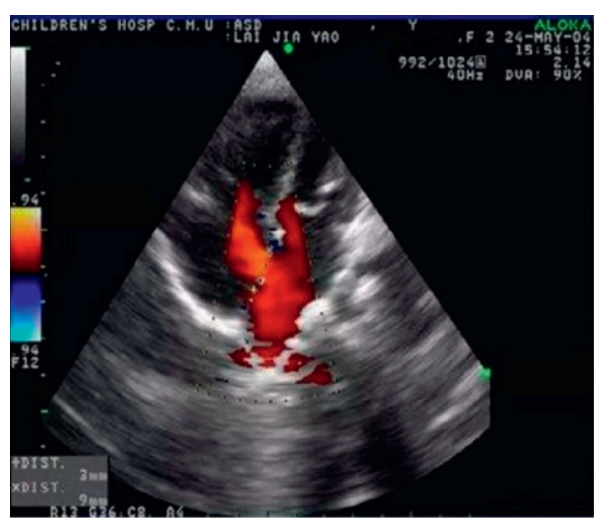

(a)

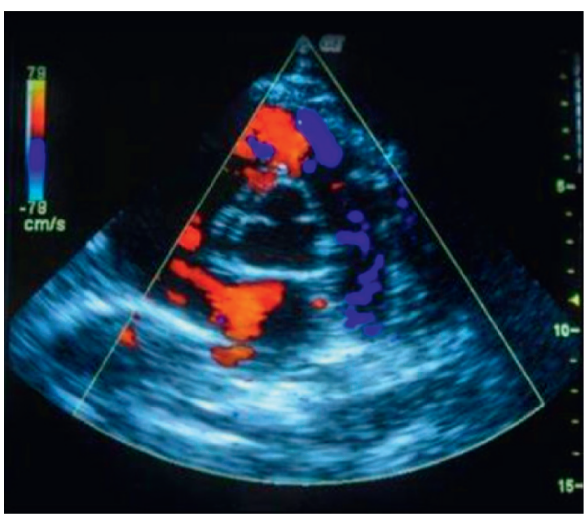

(b)

FIGURE 4: Color Doppler image of CHD.

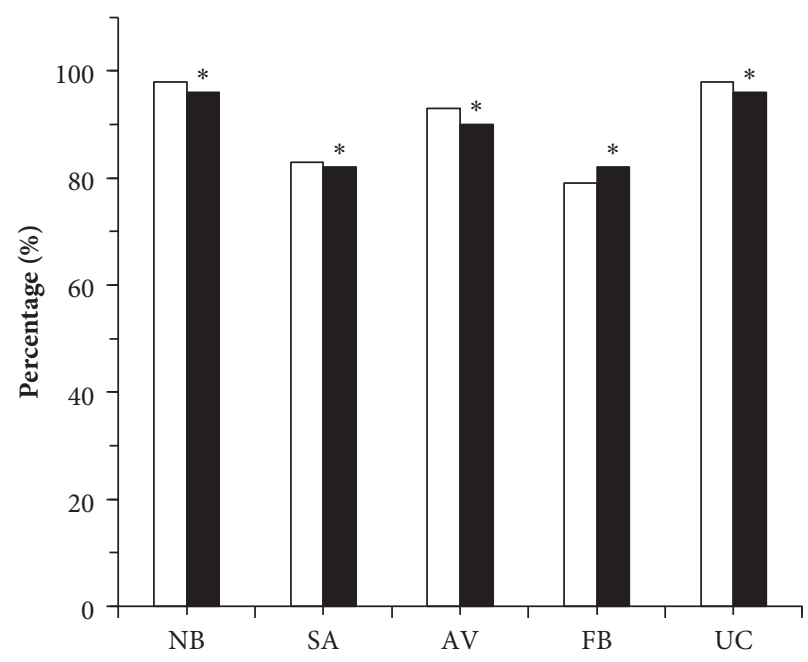

$\square \mathrm{CNN}$

- RNN

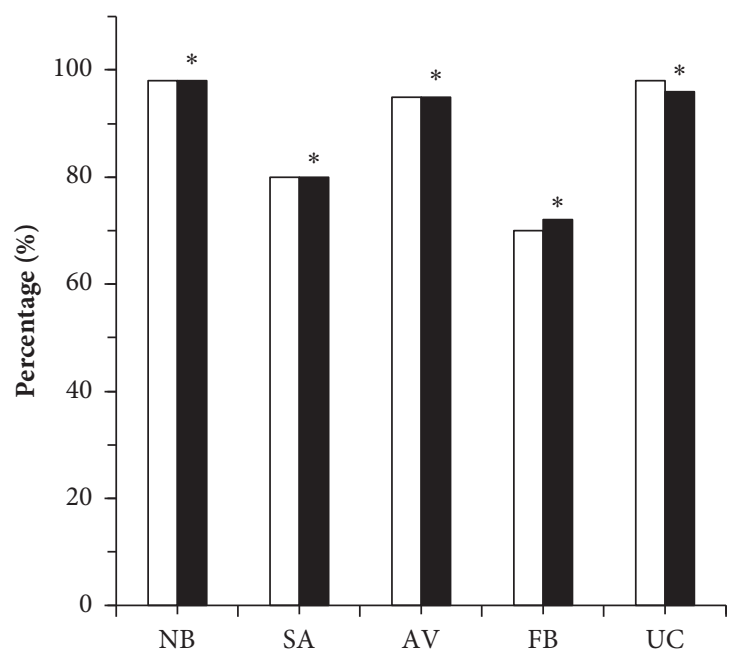

$\square \mathrm{CNN}$

- $\mathrm{RNN}$

(a)

(b)

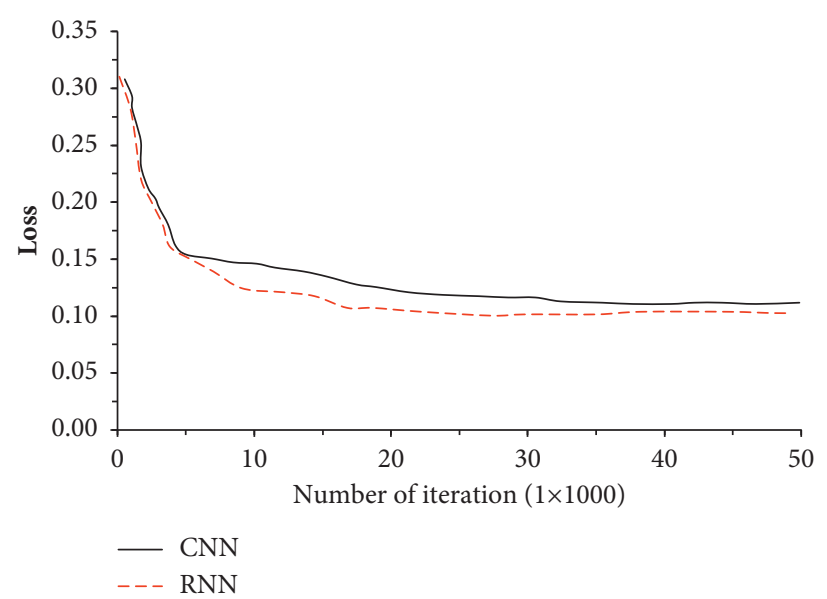

(c)

FIGURE 5: Classification results: (a) sensitivity; (b) true positive rate; (c) loss value and the number of iterations of CNN and RNN. 


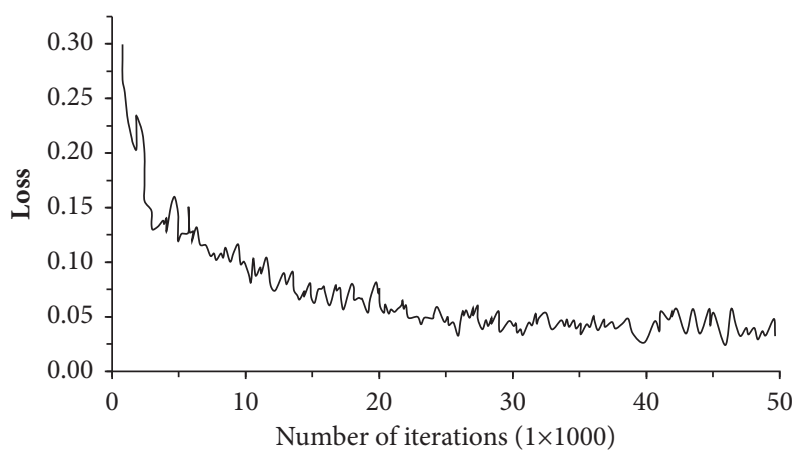

Figure 6: The loss value and the number of iterations of multimodal neural network ECG algorithm.

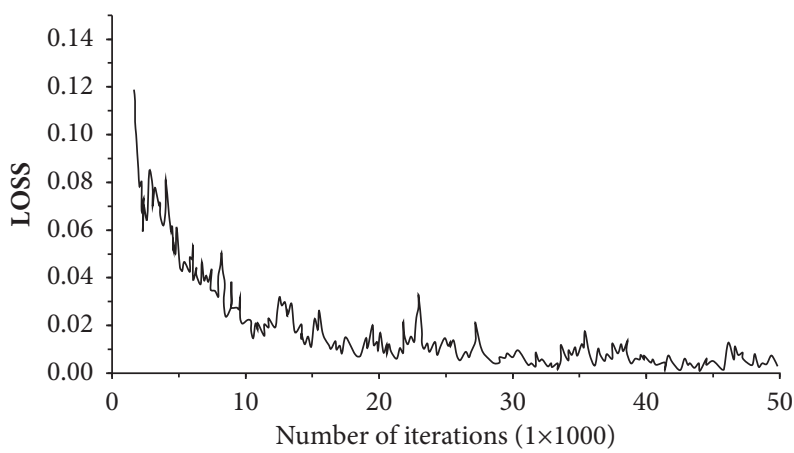

Figure 7: The loss value and number of iterations of improved multimodal neural network ECG algorithm.

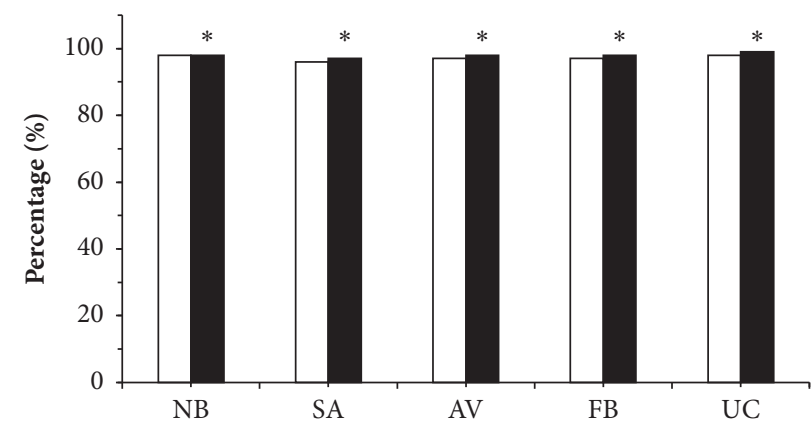

口 Multimodal Neural Network

- Improved Multimodal Neural Network

(a)

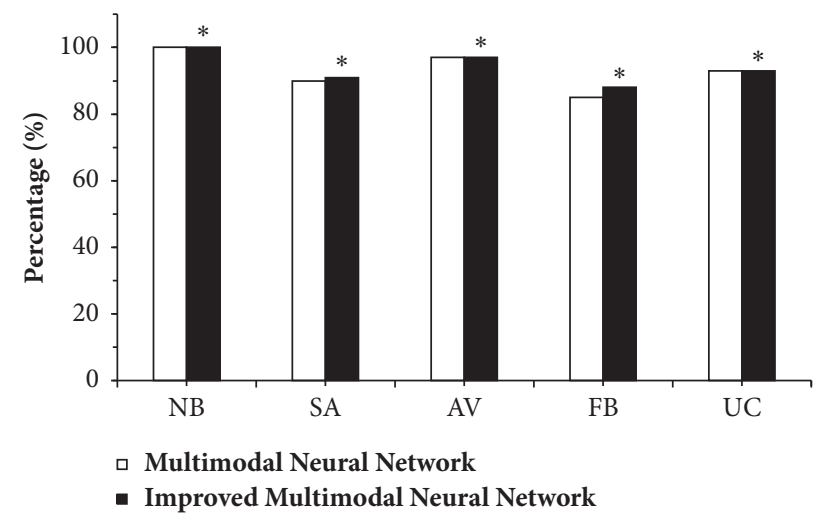

(b)

FIGURE 8: Comparison of classification results: (a) sensitivity; (b) true positive rate.

feature wave positioning on the preprocessed ECG signal and obtaining the corresponding feature wave parameters are the prerequisites for the automatic identification and classification of ECG signals [10]. The research of Patro et al. mentioned that $\mathrm{CNN}$ was used in many medical image recognitions, and the overall resolution was better [11]. It was similar to the results of ECG data classification based on the network in the study. However, the CNN does not have the ability to directly simulate the dynamic characteristics of time series data. Moreover, convolution operation in the time dimension cannot make full use of the forward and backward correlation of ECG signals in the time dimension. As a result, it is difficult for it to find effective temporal characteristics. Therefore, for the one-dimensional ECG signal that needs to be processed, modeling the sequence changes in time is needed [12-14]. Sharma et al. counted and sorted algorithms used in medical images. The results showed that LSTM based on RNN can solve the problems of overfitting, gradient dispersion, difficulty in training, and other issues in the training of neural network model [15]. It was consistent with the results in the study that classification of the BLSTM neural network obtained was better than that of the CNN. The advantages of CNN and RNN were further combined for different processing objects to propose a multimodal neural network structure. The proposed one used CNN to extract features of ECG signals and was 


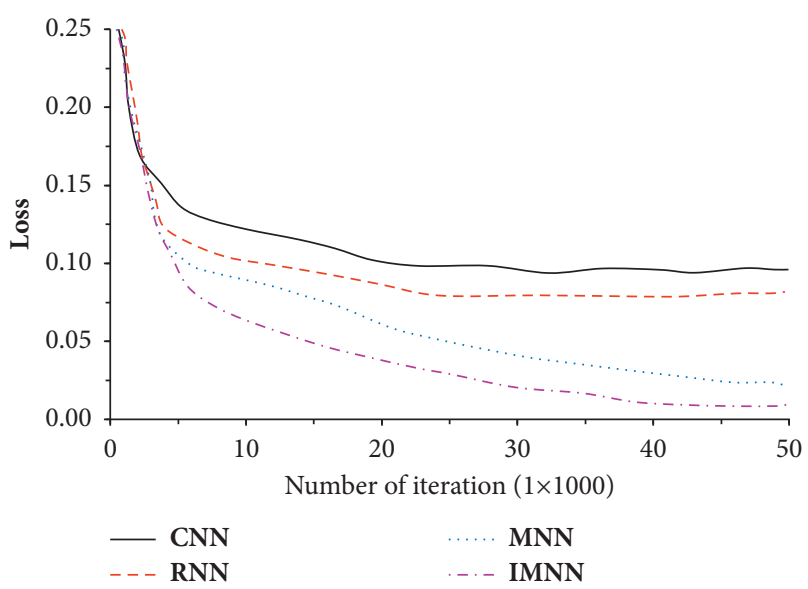

FIGURE 9: The relationship between the loss value and the number of iterations.

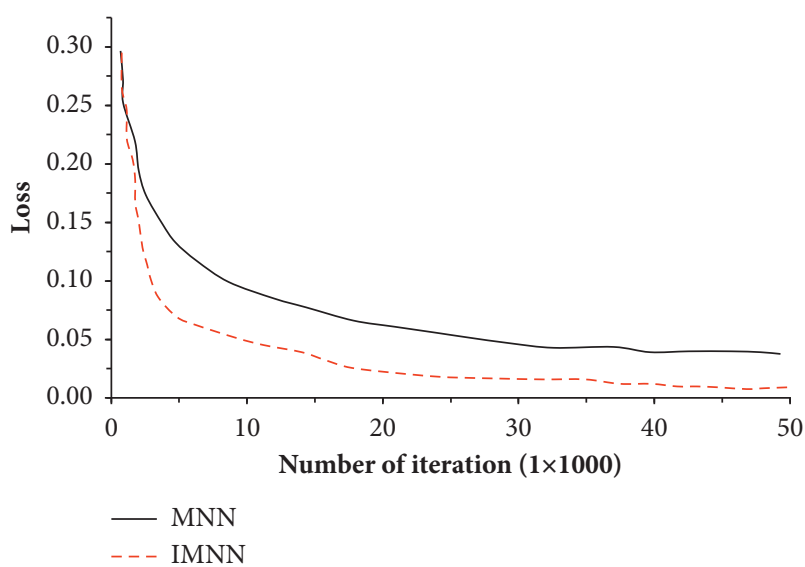

FIgURE 10: Comparison of accuracy of ECG classifications.

combined with RNN that was good at processing time series. The combined automatic identification and classification method was able to better solve the problem of poor generalization ability of the automatic classification algorithm. These problems are due to individual differences such as heartbeat intensity and heart rate in the same disease. Besides, the combined method can obviously improve the identification and classification of ECG signals. Canobbio et al. also mentioned in the research that the multimodal neural network can distinguish ECG characteristics well [16].

\section{Conclusion}

Based on the study of the single CNN ECG algorithm and the RNN ECG algorithm, the multimodal neural network ECG algorithm was constructed and corresponding improvements were made to the algorithm on this basis. Then, it was applied in the simulated environment and CHD patients, respectively. The results suggested that the multimodal neural network ECG algorithm itself has certain advantages, and the classification accuracy rate is high. On this basis, the improved multimodal neural network ECG algorithm has further improved the classification accuracy and can be applied to actual cases. In this study, a new research method of ECG of patients with CHD is proposed, and the corresponding optimization is carried out in combination with the deep neural network, which is worthy of clinical promotion. However, the research is only limited to single-lead ECG, and whether it performs well in multilead ECG images needs to be further explored. At the same time, there are fewer samples in this experimental study and there is no subdivision of patients with different types of CHD, which requires further in-depth study.

\section{Data Availability}

The data used to support the findings of this study are available from the corresponding author upon request.

\section{Conflicts of Interest}

The authors declare that they have no conflicts of interest.

\section{References}

[1] U. R. Acharya, H. Fujita, S. L. Oh et al., "Automated identification of shockable and non-shockable life-threatening ventricular arrhythmias using convolutional neural network," Future Generation Computer Systems, vol. 79, pp. 952-959, 2018.

[2] S. Kiranyaz, T. Ince, and M. Gabbouj, "Real-time patientspecific ECG classification by 1 -d convolutional neural networks," IEEE Transactions on Biomedical Engineering, vol. 63, no. 3, pp. 664-675, 2016.

[3] B. Pourbabaee, M. J. Roshtkhari, and K. Khorasani, "Deep convolutional neural networks and learning ECG features for screening paroxysmal atrial fibrillation patients," IEEE Transactions on Systems, Man, and Cybernetics: Systems, vol. 48, no. 12, pp. 2095-2104, 2018.

[4] K. K. Patro, S. P. R. Reddi, S. K. E. Khalelulla, P. Rajesh Kumar, and K. Shankar, "ECG data optimization for biometric human recognition using statistical distributed machine learning algorithm," The Journal of Supercomputing, vol. 76, no. 2, pp. 858-875, 2020.

[5] K. Hanbay, "Deep neural network based approach for ECG classification using hybrid differential features and active learning," IET Signal Processing, vol. 13, no. 2, pp. 165-175, 2018.

[6] M. Gadaleta, M. Rossi, S. R. Steinhubl et al., "Deep learning to detect atrial fibrillation from short noisy ecg segments measured with wireless sensors," Circulation, vol. 138, no. 1, Article ID A16177, 2018.

[7] S. M. Mathews, C. Kambhamettu, and K. E. Barner, "A novel application of deep learning for single-lead ECG classification," Computers in Biology and Medicine, vol. 99, pp. 53-62, 2018.

[8] J. Q. Lu, S. Wang, J. Yin et al., "A machine learning model using gut microbiome data for predicting changes of trimethylamine-N-oxide in healthy volunteers after choline consumption," Nan Fang Yi Ke Da Xue Xue Bao, vol. 37, no. 3, pp. 290-295, 2017.

[9] O. Faust, Y. Hagiwara, T. J. Hong, O. S. Lih, and U. R. Acharya, "Deep learning for healthcare applications based on physiological signals: a review," Computer Methods and Programs in Biomedicine, vol. 161, pp. 1-13, 2018. 
[10] B. Hwang, J. You, T. Vaessen, I. Myin-Germeys, C. Park, and B.-T. Zhang, "Deep ECGNet: an optimal deep learning framework for monitoring mental stress using ultra shortterm ECG signals," Telemedicine and e-Health, vol. 24, no. 10, pp. 753-772, 2018.

[11] K. K. Patro, P. R. Kumar, and P. R. Kumar, "AMachine learning classification approaches for biometric recognition system using ECG signals," Journal of Engineering Science and Technology Review, vol. 10, no. 6, pp. 1-8, 2017.

[12] M. E. Pierpont, M. Brueckner, and W. K. Chung, "Genetic basis for congenital heart disease: revisited: a scientific statement from the American heart association," Circulation, vol. 138, no. 21, pp. e653-e711, 2018.

[13] S. C. Jin, J. Homsy, S. Zaidi et al., "Contribution of rare inherited and de novo variants in 2,871 congenital heart disease probands," Nature Genetics, vol. 49, no. 11, pp. 1593-1601, 2017.

[14] F. Richter, S. U. Morton, S. W. Kim et al., "Genomic analyses implicate noncoding de novo variants in congenital heart disease," Nature Genetics, vol. 52, no. 8, pp. 769-777, 2020.

[15] A. Sharma, L. Wasson, J. Willcox et al., "Human induced pluripotent stem cells model gata6-associated congenital heart disease," Circulation, vol. 140, no. 1, Article ID A9899, 2019.

[16] M. M. Canobbio, C. A. Warnes, and J. Aboulhosn, "Management of pregnancy in patients with complex congenital heart disease: a scientific statement for healthcare professionals from the American heart association," Circulation, vol. 135, no. 8, pp. e50-e87, 2017. 